\title{
The entanglement entropy of one-dimensional gases
}

\author{
Pasquale Calabrese, Mihail Mintchev and Ettore Vicari \\ Dipartimento di Fisica dell'Università di Pisa and INFN, Pisa, Italy
}

(Dated: May 28, 2018)

\begin{abstract}
We introduce a systematic framework to calculate the bipartite entanglement entropy of a spatial subsystem in a one-dimensional quantum gas which can be mapped into a noninteracting fermion system. To show the wide range of applicability of the proposed formalism, we use it for the calculation of the entanglement in the eigenstates of periodic systems, in a gas confined by boundaries or external potentials, in junctions of quantum wires and in a time-dependent parabolic potential.
\end{abstract}

PACS numbers: 05.70.Jk,03.67.Mn,71.10.Ca

Entanglement is a fundamental phenomenon of quantum mechanics. Much theoretical work has focused on the entanglement properties of quantum many-body systems, showing their importance to characterize the manybody dynamics [1]. In particular, lots of studies have been devoted to quantify the highly nontrivial connections between different parts of an extended quantum system, by computing von Neumann or Rényi entanglement entropies of the reduced density matrix $\rho_{A}$ of a subsystem $A$. The most remarkable result is the universal behavior at 1D quantum critical points, determined by the central charge of the underlying conformal field theory (CFT) 2 25]. For a partition of an infinite 1D system into a finite piece $A$ of length $\ell$ and the remainder, the entanglement entropy for $\ell$ much larger than the short-distance cutoff $a$ is

$$
S_{1} \equiv-\operatorname{Tr}\left[\rho_{A} \ln \rho_{A}\right]=\frac{c}{3} \ln \frac{\ell}{a}+O(1),
$$

where $c$ is the central charge. This result has been confirmed in many spin-chains and in 1D itinerant systems on the lattice [1. These studies have allowed a deeper understanding of 1D simulation algorithms based on the so-called matrix product states [6]. However, the same result must be valid also in systems in continuous space (when the UV cutoff is properly imposed). Apart from the interest to describe trapped 1D gases experimentally realized with cold atoms, the entanglement of continuous models is also instrumental to develop 1D tensor network algorithms for gases, as the one proposed in [7. Despite of this fundamental interest, almost no effort (with the exception of Refs. 8, 9, and the orbital partitioning in quantum Hall states [10]) has been devoted to the spatial entanglement of gas models (that is distinguished from the particle partitioning [11]).

In this Letter we present a systematic framework to tackle free gases in any external conditions for an arbitrarily large number of particles. The most general result of this investigation is that, when dealing with a finite number of particles $N$, the 1D entanglement entropy grows like $\ln N$, with a prefactor that again is given by the central charge. In this formulation $N$ acts as an explicit UV cutoff, representing a concrete alternative to the lattice. While the $\ln N$ behavior could have been predicted also on the basis of scaling arguments, its validity in many different physical situations is not obvious a priori. Furthermore, being the method very general, it allows us to derive exact predictions that, in some cases, were not known from the lattice and/or CFT arguments. We apply our framework only to gases of spinless fermions, but its validity is more general. It is indeed straightforward to include spin degrees of freedom. Furthermore, the 1D Bose gas in the limit of strong shortranged repulsive interaction (corresponding to a gas of impenetrable bosons, describing also the dilute limit of the finite-strength model [12]) can be mapped to spinless fermions, so that their entanglement entropies coincide.

In the following we first present the general framework based on a mapping from a continuous Fredholm determinant into a standard one of dimension $N$. We then show the power of the method applying it to several physical situations, including wires junctions and non-equilibrium problems that are not yet solved on the lattice.

The method. Let us consider a system of $N$ noninteracting spinless fermions with discrete one-particle energy spectrum. As well known, the many body wave functions $\Psi\left(x_{1}, \ldots, x_{N}\right)$ can be built from the one-particle eigenstates via $\Psi\left(x_{1}, \ldots, x_{N}\right)=\operatorname{det}\left[\phi_{k}\left(x_{n}\right)\right] / \sqrt{N !}$, where the normalized wave functions $\phi_{k}(x)$ represent the occupied single-particle energy levels. The ground state is obtained by filling the lowest $N$ energy levels. Thus, the ground-state two-point correlator reads

$$
\mathbb{C}(x, y) \equiv\left\langle c^{\dagger}(x) c(y)\right\rangle=\sum_{k=1}^{N} \phi_{k}^{*}(x) \phi_{k}(y),
$$

where $c(x)$ is the fermionic annihilation operator and the one-particle eigenfunctions $\phi_{k}(x)$ are ordered according to their energies.

We want to compute the bipartite entanglement entropy of a space interval $A$, extending from $x_{1}$ to $x_{2}$, in this fermion gas. For this purpose, we introduce the Fredholm determinant

$$
D_{A}(\lambda)=\operatorname{det}[\lambda \mathbb{I}-\mathbb{C}],
$$

where the continuous matrix $\mathbb{C}$ and the identity $\mathbb{I}$ are restricted to the part at hand, i.e., from $x_{1}$ to $x_{2}$. Then, 
calculations based on the Wick theorem [13], and the integral representation of Ref. [14 for the Rènyi entanglement entropy, allow us to write

$$
S_{\alpha}\left(x_{1}, x_{2}\right) \equiv \frac{\ln \operatorname{Tr} \rho_{A}^{\alpha}}{1-\alpha}=\oint \frac{d \lambda}{2 \pi i} e_{\alpha}(\lambda) \frac{d \ln D_{A}(\lambda)}{d \lambda},
$$

where the integration contour encircles the segment $[0,1]$, and

$$
e_{\alpha}(\lambda)=\frac{1}{1-\alpha} \ln \left[\lambda^{\alpha}+(1-\lambda)^{\alpha}\right]
$$

For $\alpha \rightarrow 1$ it reduces to the von Neumann definition (1). The integral representation (4) has been already derived and used in the context of discrete chain models [14, thus involving the determinant of a standard matrix with the lattice sites as indeces.

The Fredholm determinant is turned into a standard one by introducing the $N \times N$ overlap matrix $\mathbb{A}$ (also considered in Ref. 8]) with elements

$$
\mathbb{A}_{n m}=\int_{x_{1}}^{x_{2}} d z \phi_{n}^{*}(z) \phi_{m}(z), \quad n, m=1, \ldots, N,
$$

such that $\operatorname{Tr} \mathbb{C}^{k}=\operatorname{Tr} \mathbb{A}^{k}$ for any $k$, thus

$$
\ln D_{A}(\lambda)=-\sum_{k=1}^{\infty} \frac{\operatorname{Tr} \mathbb{C}^{k}}{k \lambda^{k}}=-\sum_{k=1}^{\infty} \frac{\operatorname{Tr} \mathbb{A}^{k}}{k \lambda^{k}}=\sum_{m=1}^{N} \ln \left(\lambda-a_{m}\right),
$$

where $a_{m}$ are the eigenvalues of $\mathbb{A}$ [we use the standard relation for Fredholm determinants $\ln \operatorname{det}[\mathbb{I}-z \mathbb{M}]=$ $-\sum_{k=1}^{\infty}\left(z^{k} \operatorname{Tr} \mathbb{M}^{k}\right) / k$ and we drop off a term $\propto \ln \lambda$ giving vanishing contribution in Eq. (4)]. Inserting it into the integral (4), we obtain

$$
S_{\alpha}\left(x_{1}, x_{2}\right)=\oint \frac{d \lambda}{2 \pi i} \sum_{m=1}^{N} \frac{e_{\alpha}(\lambda)}{\lambda-a_{m}}=\sum_{m=1}^{N} e_{\alpha}\left(a_{m}\right),
$$

as a consequence of the residue theorem.

The matrix $\mathbb{A}$ is easily obtained for any non-interacting model from the one-particle wave functions, as the definition (6) shows. Calculating the entanglement entropies is then reduced to an $N \times N$ eigenvalue problem that can be easily solved numerically and in some instances even analytically, as we are going to show. Details of the calculations will be reported elsewhere.

Ground state of a periodic system. In a system of length $L$ with periodic boundary conditions (BC), the one-particle wave-functions are $\phi_{k}(x)=e^{2 \pi i k x / L} / \sqrt{L}$ with wave numbers $k \in \mathbb{Z}$. In the ground state of the fermion gas, filling the $N k$-modes with lowest energies, the matrix $\mathbb{A}$ associated with a segment of length $\ell$ is

$$
\mathbb{A}_{n m}=\frac{\sin \pi(n-m) X}{\pi(n-m)}, \quad X \equiv \ell / L,
$$

$n, m=1, \ldots, N$. By inserting the $N$ eigenvalues of $\mathbb{A}$ into Eq. 8), we obtain the entanglement entropy in a system
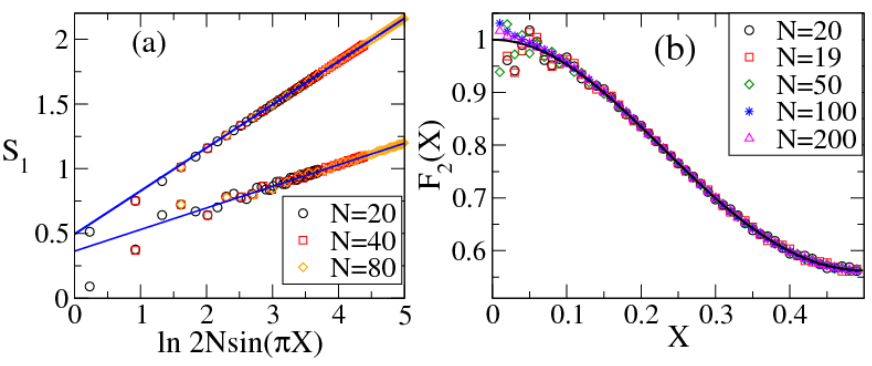

FIG. 1: (a) Entanglement entropy $S_{1}$ for periodic (uppermost set of data) and Dirichlet (bottom) BC compared with the asymptotic results for different values of $N(X=\ell / L)$. (b) The function $F_{2}(X)$ for the finite-size scaling behavior of the particle-hole excited state vs the CFT prediction [20.

of $N$ particles. Furthermore, since $\ln D_{A}=\ln \operatorname{det} \mathbb{G}$, with $\mathbb{G} \equiv \lambda \mathbb{I}-\mathbb{A}$ an $N \times N$ Toeplitz matrix, we can use the Fisher-Hartwig conjecture [15] to rigorously infer that

$$
S_{\alpha}=\frac{1}{6}\left(1+\alpha^{-1}\right) \ln (2 N \sin \pi X)+b_{\alpha}+O\left(N^{-\frac{2}{\alpha}}\right),
$$

where $b_{\alpha}$ and the leading $O\left(N^{-\frac{2}{\alpha}}\right)$ corrections can also be computed analytically [16]. Fig. 1] shows a comparison with exact finite- $N$ calculations, for $\alpha=1$. Eq. 10 agrees with the CFT prediction for finite systems [4, obtained from Eq. (1) replacing $\ell$ with the chord length $L / \pi \sin (\pi X)$. Thus, Eq. 10 represents the first explicit analytic confirmation of this CFT prediction.

Systems with hard-wall potential. We now consider a gas of spinless fermions confined in the interval $[0, L]$ by a hard-wall potential (i.e., the gas density vanishes for $x \notin[0, L])$. We consider a bipartition starting from the boundary, i.e., $A=[0, \ell]$, but the general case can also be treated. Some algebra similar to before leads to

$$
\mathbb{A}_{n m}=\mathbb{B}_{n m}(X) \equiv \frac{\sin [\pi(n-m) X]}{\pi(n-m)}-\frac{\sin [\pi(n+m) X]}{\pi(n+m)} .
$$

Using a recent generalization of the Fisher-Hartwig conjecture to Toeplitz+Henkel matrices [16, 17, we obtain

$$
S_{\alpha}=\frac{1}{12}\left(1+\alpha^{-1}\right) \ln (4 N \sin \pi X)+\frac{b_{\alpha}}{2}+O\left(N^{-\frac{1}{\alpha}}\right)
$$

A comparison of the finite- $N$ results with Eq. 12 is shown in Fig. 1. The leading oscillating $O\left(N^{-\frac{1}{\alpha}}\right)$ corrections can also be computed. They correspond to the $O\left(\ell^{-\frac{1}{\alpha}}\right)$ corrections found within CFT [18, 19.

Excited states can be easily treated in this formalism by summing in Eq. (2) over the occupied one-particle states representing the excited states. As an example, let us consider the particle-hole excitation in a periodic system obtained by moving one particle from the highest occupied level to the first available one. The corresponding $N \times N$ matrix $\mathbb{A}$ has the first $n-1$ raws and columns identical to the ground state, and the last 
different. Note that it ceases to be a Toeplitz matrix. In Fig. 1 we compare the scaling function $F_{2}(X) \equiv$ $\exp \left[S_{2}-1 / 4 \ln (2 N \sin \pi X)-b_{2}\right]$ for several values of $N$ with the corresponding CFT prediction [20] $F_{2}(X)=$ $[7+\cos (2 \pi X)]^{2} / 64$. The CFT curve is clearly approached in the large- $N$ limit.

Star graphs or wire-junctions. A novel application of the outlined method consists in determining the entanglement at a junction of quantum wires (called also star graph). Networks of quantum wires with junctions attracted recently a lot of attention [21, mainly because of their possible applications in nanocircuits. A star graph consists of $M$ wires joining in a single point (called vertex). We consider wires whose bulk is described by noninteracting spinless fermions. The only interaction is localized at the vertex and it is encoded in a $M \times M$ scattering matrix $\mathbb{S}$ 21, 22, as pictorially showed in Fig. 2. We consider wires of finite length $L$ with hard-wall boundary conditions at their ends. Scale invariant junctions can be constructed and classified [21, 22]: they are either isolated points or families with free parameters.

Let us first consider the case of two wires, i.e., a gas of free fermions with a localized impurity at the vertex. The allowed scale invariant conditions at the vertex are [22]: the two trivial ones (Dirichlet and Neumann) which disconnect the two wires and give no entanglement, and the one-parameter family described by the scattering matrix

$$
\mathbb{S}(\epsilon)=\frac{1}{1+\epsilon^{2}}\left(\begin{array}{cc}
-1+\epsilon^{2} & 2 \epsilon \\
2 \epsilon & 1-\epsilon^{2}
\end{array}\right) .
$$

(A phase can be added, but it does not enter any measurable quantity and will be omitted.) Note that $\epsilon=1$ corresponds to full transmission, i.e., no impurity.

In order to derive the ground-state entanglement entropy of one wire, we compute the matrix $\mathbb{A}$ in Eq. 6 by diagonalizing the one-particle problem. We obtain

$$
\begin{aligned}
& \mathbb{A}_{n m}=\frac{2 \epsilon}{1+\epsilon^{2}} \mathbb{B}_{n m}(1 / 2) \quad \text { for } n \neq m \\
& \mathbb{A}_{n n}=\frac{1}{1+\epsilon^{2}} \text { for odd } n, \quad \mathbb{A}_{n n}=\frac{\epsilon^{2}}{1+\epsilon^{2}} \text { for even } n
\end{aligned}
$$

where $\mathbb{B}_{n m}$ is defined in Eq. (11). Then, we determine the wire entanglement entropy $S_{\alpha}(s ; N)$ as a function of the transmission coefficient

$$
s \equiv \frac{2 \epsilon}{1+\epsilon^{2}} .
$$

We observe a logarithmic growth

$$
S_{\alpha}(s ; N)=\mathcal{C}_{\alpha}(s) \ln N+O(1),
$$

with a prefactor $\mathcal{C}_{\alpha}(s)$ that depends on $s$ and not only on the central charge, see Fig. 2. Assuming universality in terms of $s$, we can exploit the results of Ref. 23] for the lattice Ising and XX models with a defect, to infer

$$
\mathcal{C}_{\alpha}(s)=\frac{2}{\pi^{2}(1-\alpha)} \int_{0}^{\infty} d x \ln \left[\frac{1+e^{-2 \alpha \omega(s)}}{\left(1+e^{-2 \omega(s)}\right)^{\alpha}}\right],
$$
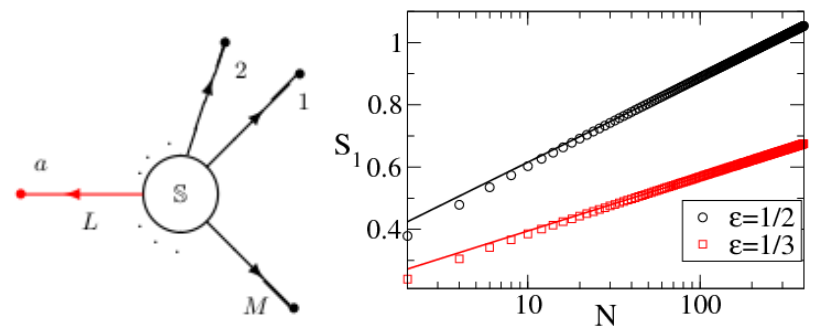

FIG. 2: Left: $M$ wires interacting through the scattering matrix $\mathbb{S}$. Right: Entanglement entropy $S_{1}$ for a junction of two wires and for different values of the transmission coefficient $s$. The full lines correspond to the asymptotic behavior 16 . with $\mathcal{C}_{1}(s)$ given by the limit $\alpha \rightarrow 1$ of Eq. 17 .

where $\omega(s)=\operatorname{acosh}[(\cosh x) / s]$. The perfect agreement, see Fig. 2, with the large- $N$ behavior of our numerical evaluations nicely confirms the universality conjecture.

The case of a general number of wires $M$ can be analogously treated. After diagonalizing the model 22, we construct the correlation matrix reduced to one wire $a$, and then the $2 N / M \times 2 N / M$ matrix $\mathbb{A}$ (for simplicity we assume $N$ to be a multiple of $M$ ) corresponding to the same wire. After some algebra we obtain

$$
\begin{aligned}
& \mathbb{A}_{n m}=\Upsilon_{a} \sqrt{1-\Upsilon_{a}^{2}} \mathbb{B}_{n m}(1 / 2) \quad \text { for } n \neq m, \quad \\
& \mathbb{A}_{n n}=\Upsilon_{a}^{2} \text { for even } n, \quad \mathbb{A}_{n n}=\left(1-\Upsilon_{a}^{2}\right) \text { for odd } n,
\end{aligned}
$$

where $\Upsilon_{a}^{2}=\left(1+\mathbb{S}_{a a}\right) / 2$. Notice that $\mathbb{A}$ has the same form of that for the two-wire problem, cf. Eq. (14), which is recovered setting $\Upsilon_{a}=\epsilon / \sqrt{1+\epsilon^{2}}$. Thus, using Eq. (16), we also derive the asymptotic behavior of the entanglement entropy of any of the $M$ wires given by Eq. (16) with $s=2 \Upsilon_{a} \sqrt{1-\Upsilon_{a}^{2}}=\sqrt{1-\mathbb{S}_{a a}^{2}}$. Note that $s^{2}$ is the total probability of a signal to be transmitted from the wire $a$ to all other wires. Being $\mathcal{C}_{\alpha}$ a monotonous function of $s$, the maximum entanglement entropy is always obtained for $s=1\left(\mathbb{S}_{a a}=0\right)$, i.e., in the absence of reflection.

Non-equilibrium evolution. All results presented so far concern the eigenstates of free-fermionic Hamiltonians. However, the method allows us to also obtain numerically exact and/or analytic computations out of equilibrium. Indeed Eq. 2 is valid if we replace the one-particle eigenfunctions $\phi_{k}(x)$ with appropriate solutions of the corresponding time-dependent one-particle Schrödinger equation. As an example that can be treated analytically, we consider the off-equilibrium quantum dynamics of a gas in a time-dependent harmonic potential.

Using the method outlined above and the knowledge of the eigenstates of the harmonic oscillator, one can straightforwardly calculate the ground-state (equilibrium) entanglement entropy of any spatial subsystem $\left[x_{1}, x_{2}\right]$. In particular, the half-space entanglement entropy, i.e., of $[-\infty, 0]$, behaves as $S_{\alpha}(-\infty, 0)=\frac{1}{12}(1+$ $\left.\alpha^{-1}\right) \ln N+e_{\alpha}+O\left(N^{-\frac{1}{\alpha}}\right)$, which can be analytically obtained, including the constant $e_{\alpha}$, by developing results 
of Ref. 24. In the presence of a time-dependent potential $V(x)=\frac{1}{2} \kappa(t) x^{2}$, and starting from the ground state for a given $\kappa_{0}=\kappa(t=0)$, we can exploit the solution of the one-particle problem [25, 26] to infer that the timedependent entanglement entropy behaves as

$$
S_{\alpha}\left(x_{1}, x_{2} ; t\right)=S_{\alpha}\left(x_{1} / s(t), x_{2} / s(t) ; 0\right),
$$

where $s(t)$ is an analytical function of the time-dependent potential with $s(0)=1$. This shows the remarkable property that the evolution in a harmonic potential simply corresponds to a global rescaling of the system size. In the case of a quantum quench with an instantaneous removal of the harmonic potential of frequency $\sqrt{\kappa_{0}}$, we have $s(t)=\sqrt{1+\kappa_{0} t^{2}}$. Then for $t \rightarrow \infty, s(t)$ diverges and the entanglement of any finite interval vanishes in the long time limit, while the entanglement of any semi-infinite piece $[-\infty, x]$ tends asymptotically to $S_{\alpha}(-\infty, 0 ; 0)$.

Other non-equilibrium situations such as local quantum quenches in junctions of quantum wires (e.g., instantaneously turning on/off the point contact at the vertex) can also be tackled within this framework. They may provide important insights in view of the recent proposals of using the full counting statistics after a quench as an experimental probe and a measure of entanglement [27].

Discussions. In this Letter we have introduced a general framework to calculate the entanglement entropy of gases that have a representation in terms of free fermions. The asymptotic entanglement entropy grows like $\ln N$, with a prefactor that is given by the central charge and that (in known cases) agrees with universal predictions. The UV regularization given by the number of particles $N$ is alternative to the lattice spacing and, for the description of gases, it has an immediate physical interpretation. Moreover, the formulas of the universal features of the entanglement are easier to handle in the continuum at finite $N$ than on the lattice.

We conclude by mentioning some possible extensions of this work beyond the straightforward (but still interesting) applications to other 1D free gases. Motivated by recent experiments in cold atoms, it is interesting to consider 1D gases in an external random potential that, in the case of a perfect gas, can be treated with our method. All the results we have presented are in $1 \mathrm{D}$, but the application of the method to higher dimensions is straightforward.

[1] L. Amico, R. Fazio, A. Osterloh, and V. Vedral, Rev. Mod. Phys. 80, 517 (2008); J. Eisert, M. Cramer, and M. B. Plenio, Rev. Mod. Phys. 82, 277 (2010).

[2] C. Holzhey, F. Larsen, and F. Wilczek, Nucl. Phys. B 424, 443 (1994).

[3] G. Vidal, J. I. Latorre, E. Rico, and A. Kitaev, Phys. Rev. Lett. 90, 227902 (2003).
[4] P. Calabrese and J. Cardy, J. Stat. Mech. P06002 (2004).

[5] P. Calabrese and J. Cardy, J. Phys. A 42, 504005 (2009).

[6] J.I. Cirac and F. Verstraete, J. Phys. A 42, 504004 (2009).

[7] F. Verstraete and J.I. Cirac, Phys. Rev. Lett. 104, 190405 (2010).

[8] I. Klich, J. Phys. A 39, L85 (2006).

[9] F.N.C Paraan et al., 1105.1211.

[10] M. Haque, O. Zozulya, and K. Schoutens, Phys. Rev. Lett. 98, 060401 (2007); H. Li and F. D. M. Haldane, Phys. Rev. Lett. 101, 010504 (2008).

[11] M. Haque, O. Zozulya, K. Schoutens, J. Phys. A 42, $504012(2009)$.

[12] E.H. Lieb and W. Liniger, Phys. Rev. 130, 1605 (1963).

[13] I. Peschel and V. Eisler, J. Phys. A 42, 504003 (2009).

[14] B-Q Jin and V.E. Korepin, J. Stat. Phys. 116, 79 (2004).

[15] The Fisher-Hartwig conjecture [M. E. Fisher and R. E. Hartwig, Adv. Chem. Phys. 15, 333 (1968)] has been rigorously proven for the case at hand, see e.g. E. L. Basor and K. E. Morrison, Lin. Alg. Appl. 202, 129 (1994).

[16] The matrices (9) and (11) have the same forms of the two-point correlation functions of lattice free fermions in the thermodynamic limit without 14 or with boundaries [19]. On the lattice, the indices are related to the sites, with the Fermi momentum $k_{F}$ replacing $\pi \ell / L$. This correspondence allows us to make use of all results for the asymptotic entanglement entropy, with $N$ playing the role of the size $\ell$ of the subsystem. In particular, the results reported in Ref. [19, 28, derived using the Fisher-Hartwig conjecture, allows us to compute also the oscillating $O\left(N^{-\frac{2}{\alpha}}\right)$ and $O\left(N^{-\frac{1}{\alpha}}\right)$ corrections.

[17] P. Deift, A. Its, and I. Krasovsky, 0905.0443.

[18] J. Cardy and P. Calabrese, J. Stat. Mech. P04023 (2010).

[19] M. Fagotti and P. Calabrese, J. Stat. Mech. P01017 (2011).

[20] F. C. Alcaraz, M. I. Berganza, and G. Sierra, Phys. Rev. Lett. 106, 201601 (2011).

[21] C. Nayak, M.P.A. Fisher, A.W.W. Ludwig, H.H. Lin, Phys. Rev. B 59, 15694 (1999); C. Chamon, M. Oshikawa, I. Affleck, Phys. Rev. Lett. 91, 206403 (2003); M. Oshikawa, C. Chamon, I. Affleck, J. Stat. Mech. P02008 (2006).

[22] B. Bellazzini and M. Mintchev, J. Phys. A 39, 11101(2006); B. Bellazzini, M. Mintchev, and P. Sorba J. Phys. A 40, 2485 (2007); B. Bellazzini, P. Calabrese, M. Mintchev, Phys. Rev. B 79, 085122 (2009).

[23] V. Eisler and I. Peschel, Ann. Phys. (Berlin) 522, 679 (2010).

[24] M. Campostrini and E. Vicari, J. Stat. Mech. (2010) P08020; Phys. Rev. A 81, 063614 (2010).

[25] M. Campostrini and E. Vicari, Phys. Rev. A 82, 063636 (2010).

[26] M.D. Girardeau and E.M. Wright, Phys. Rev. Lett. 84, 5691 (2000).

[27] I. Klich and L. Levitov, Phys. Rev. Lett. 102, 100502 (2009); B. Hsu, E. Grosfeld, and E. Fradkin, Phys. Rev. B 80, 235412 (2009); J. Cardy, Phys. Rev. Lett. 106, 150404 (2011).

[28] P. Calabrese, M. Campostrini, F. Essler, and B. Nienhuis, Phys. Rev. Lett. 104, 095701 (2010); P. Calabrese and F. H. L. Essler, J. Stat. Mech. (2010) P08029. 